\title{
Relationship between air traffic demand, safety and complexity in high density airspace in Europe
}

\author{
Tamara Pejovic ${ }^{1, *}$, Fedja Natjasov $^{2}$, and Dusan Crnogorac ${ }^{3}$ \\ ${ }^{1}$ EUROCONTROL, Performance Review Unit, Belgium \\ ${ }^{2}$ University of Belgrade, Faculty of Transport and Traffic Engineering, Division of Airports and Air \\ Traffic Safety, Serbia \\ ${ }^{3}$ University of Belgrade, Faculty of Transport and Traffic Engineering, Computer Center, Serbia
}

\begin{abstract}
Air traffic performance of the European air traffic system depends not only on traffic demand but also on airspace structure and its traffic distribution. These structural (airspace structure) and flow characteristics (factors such as traffic volume, climbing/descending traffic, mix of aircraft type, military area activity) influence airspace complexity, which can affect controller workload and influence the probability of safety occurrence. In other words, all these dynamic and static complexity components can potentially have an impact upon the safety of the air traffic management (ATM) system. Having in mind fluctuation in traffic on daily, seasonal or annual level in certain airspace, a few questions arise: How changes in traffic demand influence complexity and conflict risk? Is there any correlation between traffic demand, conflict risk and complexity? Are there any differences between seasons? For that purpose, an investigation is performed on FAB Europe Central (FABEC) airspace, based on two weeks of operated traffic during the summer and winter of 2017. Air traffic complexity is estimated using the EUROCONTROL complexity metrics, while conflict risk is assessed using the conflict risk assessment simulation tool. Results show that certain positive relationship exists between traffic demand, conflict risk and complexity.
\end{abstract}

\section{Introduction}

In 2018, instrument flight rule (IFR) movements within the European airspace continued to grow strongly (4.65\% versus 2017), making last year a new record year in terms of traffic volumes: the number of flights controlled reached an all-time record of more than 11 million [1]. The forecast growth indicates that by 2021, the European sky will handle over 12.3 million operations. This is an incredible challenge for the safety, the en route sector capacity and impact on the environment. The implementation of two operational concepts, the free route airspace (FRA) and functional airspace block (FAB), is seen as crucial 'tools' for solving those issues.

\footnotetext{
* Corresponding author: tamara.pejovic@eurocontrol.int
} 
By definition, FRA is a specified airspace wherein users can freely plan a route between a defined entry point and a defined exit point, with the possibility of routing via intermediate (published or unpublished) waypoints, without reference to the air traffic service (ATS) route network, subject of course to availability. Within such airspace, flights remain subject to air traffic control (ATC) for the separation provision and flight level (FL) change authorizations.

The overall benefits of free route operations are distance and flight timesaving, resulting in less fuel consumption and a notable reduction of engine emissions, which benefits the environment [2]. FRA is seen as a cornerstone to improve FAB Europe Central (FABEC) structure and utilisation. From the other side, an implementation of FABs should bring further efficiency of airspace operations because FABs are 'based on operational requirements and established regardless of State boundaries, in which the provision of air navigation services and related ancillary functions are optimized and/or integrated' [3]. Currently, there are nine FABs established to cover almost the whole European airspace [3]. However, their implementation is still too slow (according to the European Commission [3]) causing inefficiency in the European ATM system.

\subsection{Complexity of air traffic}

Complexity of air traffic can be defined as the level of either perceived or actual spatial and time-related interactions between aircraft operating in a given airspace during a given period [4]. Specifically, complexity of air traffic in a given airspace can be very high solely because of the traffic intensity and its pattern in terms of mutual interactions between different traffic flows, as well as between individual aircrafts. Such presumably high complexity could be used for both planning and operational purposes mainly aimed at reducing it. Consequently, it may be reduced at the strategic, tactical and pre-tactical level. At each of these levels, it can have a spatial-based nature (such as airspace and airfield system design and/or assignment such as air routes, sectors, terminals, runway systems, etc.) and also time-based solutions (such as schedules, slot allocations, flow management, etc.). In that context, according to Netjasov et al. [4], complexity is understood as a demand characteristic of air traffic that is to be served by an appropriate supply system.

Traffic complexity affects control task complexity (Figure 1), where the control is performed by human operator [4]. It is expected that a more complex task will produce a higher workload. However, the workload influenced by task complexity differs between ATCos (Figure 1) due to differences in their working environment, perception of the traffic situation, personal experience, etc. Therefore, complexity represents a contributing factor of task complexity and ATCo workload.
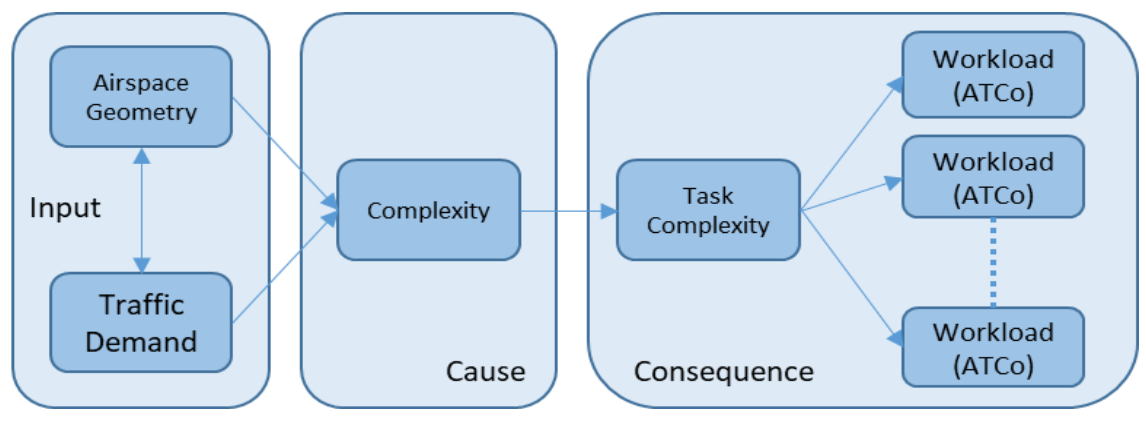

Figure. 1. Scheme of the relationship between complexity, task complexity and workload 
The approach presented in this chapter is based on EUROCONTROL [5] methodology, with exclusion of ATCo workload issue from the explicit consideration. Approach is taking a macroscopic view, and it is considering four complexity components: adjusted density, potential vertical interactions, potential horizontal interactions and potential speed interactions. A single metric, 'complexity score', which incorporates these four separate parameters, was considered as the simplest for benchmarking purposes. Recently, Pejovic and Lazarovski [6] have studied the performance of the North European Free Route Airspace using EUROCONTROL approach.

\subsection{Conflict risk}

The International Civil Aviation Organisation (ICAO) has developed the Collision Risk Model (CRM) as a mathematical tool used in predicting the risk of mid-air collision [7]. Although aircraft collisions have actually been very rare events, contributing to a very small proportion of the total fatalities, they have always caused relatively strong impact mainly due to relatively large number of fatalities per single event and occasionally the complete destruction of the aircraft involved.

From other side, one of the principal matters of concern in the daily operation of civil aviation is the prevention of conflicts, i.e. Potential Losses of Separation (PLoS) between aircraft either while airborne or on the ground, which might escalate to collisions. A PLoS is a situation when two aircrafts come closer to each other than a specified minimum distance both in the horizontal and the vertical planes. One can imagine that PLoSs are more frequent event than collisions, so assessment of conflict risk is becoming important.

In order to determine whether or not PLoS situation exists and to calculate a conflict risk value, a cylinder-shaped 'forbidden volume' is defined around the aircraft [8]. A PLoS exists between two aircrafts if one of them enters the other's forbidden volume. They could be of a crossing or an overtaking type, depending on the aircraft trajectory relations both in horizontal and vertical planes [9].

Dealing with a conflict risk instead of a collision risk (a concept established by ICAO) is enabling a proactive safety approach, which is much closer to everyday ATCo activities.

\section{Study approach}

To analyse how future changes in airspace structure and traffic flow could influence complexity and safety performance, this paper proposes a showcase methodology on the analysis of FABEC. FABEC, which includes airspaces of six countries (Belgium, France, Germany, Luxembourg, the Netherlands and Switzerland) is one of the biggest Functional Airspace Blocks in Europe and is handling more than half of the European annual traffic. According to [10] this 'airspace is one of the busiest and most complex in the world' with 'most major European airports, major civil airways and military training areas located in this area'. Since June 2013, FABEC is officially in operation.

\subsection{Traffic data and scenarios}

Two traffic scenarios covered one week of summer (July 3-9, 2017, with 131.268 flights) and winter (November 13-17, 2017, with 94.947 flights). For each traffic scenario, calculation of complexity parameters (calculated using the NEST tool [11]) and safety indicators (calculated using the Conflict Risk Assessment Tool [9]) was done using the same input - summer and winter traffic. 


\subsection{Assessment of complexity and safety indicators}

The assessment of complexity was done using the EUROCONTROL complexity score [5] as airspace complexity indicator. The two main metrics that define the complexity score are the "adjusted density" and the "structural index". The latter is derived from three parameters describing potential number of interactions in specific situations classified as vertical, horizontal and the mix of aircraft performances. The "adjusted density" assesses the potential interactions resulting from density, including uncertainty in the trajectories and time, while the "structural index" balances the density metrics according to the interaction geometry and aircraft performance differences. Formulas used for the calculation of complexity score are explained in [5].

The assessment of safety indicators was done using Conflict Risk Assessment Tool [9]. This tool is intended for the simulation of planned or analysis of realized air traffic, consisting of flight trajectories (4D trajectories) crossing a given airspace, with the aim of assessing safety performance. Conflict Risk Assessment Tool has been developed as a network based simulation model consisting of three modules, each being used for the calculation of certain safety indicators [9]: Separation violation detection module (dynamic 3D conflict detection model based on known flight intensions and distance-based separation minima - used for the calculation of duration, severity and number of PLoS), Traffic collision avoidance system activation module and Conflict risk assessment module. The safety minima separations used in analysis were: horizontal separation $-5 \mathrm{NM}$ and vertical separation - $1000 \mathrm{ft}$; however those values were relaxed for $10 \%$ (4,5 NM and $900 \mathrm{ft}$ ) in order to deal with potential position and altitude inaccuracies.

\section{Results}

\subsection{Complexity and safety indicators overall analysis}

Daily fluctuations of both complexity and safety indicators follow similar pattern throughout the week in both summer and winter. Traffic values indicate that traffic demand during winter is lower than during summer in a range from 22 to $37 \%$. Similarly the complexity score values fluctuate - summer values are higher in a range between 17 and 29\%. Overall, changes of daily complexity values are following the changes of daily traffic demand.

Contrary, the changes in daily number of PLoS and conflict risk do not follow strictly the changes in daily traffic demand. However, variations in the number of PLoS are following the changes in conflict risk. The number of PLoS during winter is lower in a range from 33 to $63 \%$. Similarly, the conflict risk shows lower values in winter in a range from 28 to $65 \%$.

Complexity analysis shows that total hours of interactions (bars, Figure 2 up) increase during winter mainly due to increase in speed interactions (yellow bars, Figure 2 up) which could indicate the greater changes in the mix of aircraft used. However, overall complexity score reduces during winter by $15-20 \%$ depending on the day of the week. This indicates that overall complexity score (black line, Figure 2 up) is mainly influenced by changes in adjusted density (green line, Figure 2 up). Adjusted density assesses aircraft interactions resulting from density, including uncertainty in the trajectories and time. Adjusted density shows that interactions are not only related with the traffic volume, however also with how this traffic is dispersed in airspace. 
The analysis of number of interactions and number of PLoS per hour of flight (Figure 2 down) shows that the total number of interactions per hour of flight reduces during winter by over $23 \%$ (0.224 in summer vs. 0.172 in winter). The number of PLoS per hour of flight is somewhat more stable and does not change much with decrease in traffic. Overall change is approximately $13 \%$ (0.015 in summer vs. 0.013 in winter).
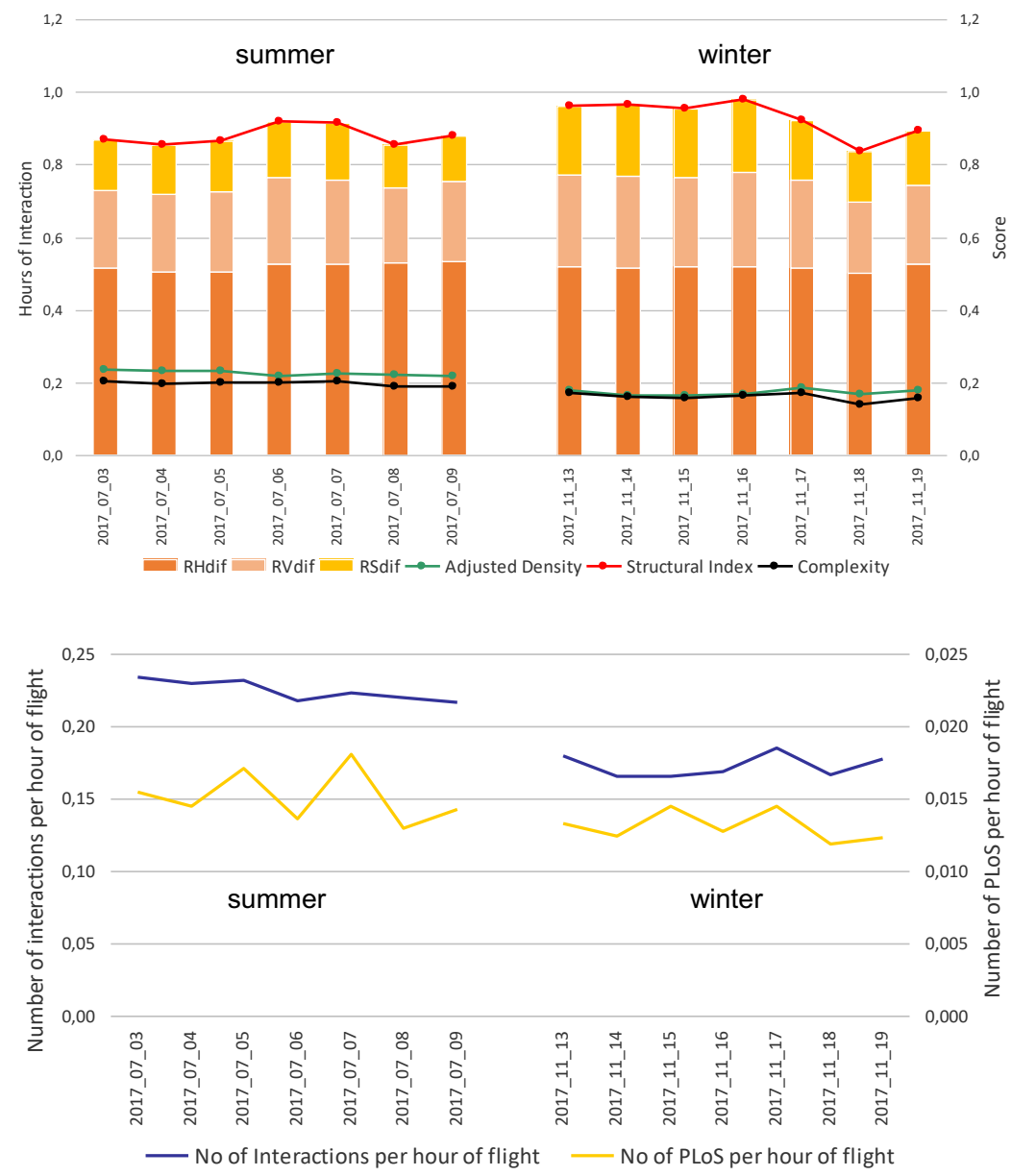

Figure. 2. Complexity parameters (up) and number of aircraft interactions and PLoS per hour of flight (down)

\subsection{Correlation between traffic, complexity and safety indicators}

A very strong correlation $\left(\mathrm{R}^{2}=0.9807\right)$ was found between the daily number of flights and complexity (Table 1). Strong correlations were also found between safety indicators (slightly better correlation with the number of PLoS) and the total number of flights. Similarly, strong correlations exist between safety indicators and complexity.

These findings could lead to a conclusion that with increase in traffic, one can expect the higher complexity, followed by the higher number of PLoS and conflict risk. In other words, this means that ATCo task load will increase, leading to a higher ATCo workload. 
Table 1. Linear correlation coefficients for both seasons combined

\begin{tabular}{|l|c|c|c|}
\hline Both seasons & Complexity & PLoS & Conflict Risk \\
\hline \# Flights & +0.9807 & +0.8819 & +0.8008 \\
\hline Complexity & - & +0.9138 & +0.8296 \\
\hline
\end{tabular}

Seasonal comparison - the results of correlation analysis between traffic demand, complexity and safety indicators (conflict risk) show the positive correlation between the number of flights and complexity score (as dependent variable) that is stronger in winter (summer $\mathrm{R}^{2}=0.7163$ (Table 2) vs. winter $\mathrm{R}^{2}=0.9022$ (Table 3)). This is expected as daily complexity scores follow the daily number of flights. Moreover this could be explained by the fact that during the winter traffic is more uniform, while during summer there are more charter and business flights (unscheduled flights) that could change traffic flows and locations of PLos points, which in turn is decreasing predictability and increasing complexity score. Operationally, this could also potentially increase ATCo's workload during summer.

Table 2. Linear correlation coefficients for summer season

\begin{tabular}{|l|c|c|c|}
\hline Summer & Complexity & PLoS & Conflict Risk \\
\hline \# Flights & +0.7163 & +0.3716 & +0.3114 \\
\hline Complexity & - & +0.6980 & +0.5144 \\
\hline
\end{tabular}

Table 3. Linear correlation coefficients for winter season

\begin{tabular}{|l|c|c|c|}
\hline Winter & Complexity & PLoS & Conflict Risk \\
\hline \# Flights & +0.9022 & +0.5005 & +0.2207 \\
\hline Complexity & - & +0.5640 & +0.2843 \\
\hline
\end{tabular}

Moreover, the positive correlation between the number of flights and conflict risk (as dependent variable) is not strong (in both seasons $\mathrm{R}^{2}<0.5$, although in summer is somewhat stronger). Similarly, the positive correlation between complexity score and conflict risk is not strong $\left(\mathrm{R}^{2}\right.$ is higher in summer than in winter).

Correlation between the number of flights and number of PLoSs is not strong (although somewhat higher in winter). Contrary, correlation between complexity scores and the number of PLoS shows a positive correlation (stronger in summer).

Overall, it can be concluded that correlation between complexity and the number of PLoSs is stronger than between complexity and conflict risk (Tables 2 and 3). Similar behaviour can be observed in the case of correlation between the number of flights and the number of PLoS. However, it has to be noted that the conflict risk as an indicator contains more information about the loss of separation than just the total number.

\subsection{Analysis of potential losses of separation}

\subsubsection{PLoS duration and severity}

Each PLoS is characterised by the combination of severity (related to the breach of separation) and duration. The severity of the PLoS depends on the minimum distance (spacing) between the pair of aircraft $\left(\mathrm{S}_{\mathrm{min}}\right)$ and the applied separation minima $\left.\left(\mathrm{Sep}_{\min }\right)\right)$. The severity presents the level of aircraft proximity and is defined either for the violation of separation in the horizontal or the vertical plane, or both [8]: Severity $=\left(S_{e p} \min -S_{\min }\right) / S_{\text {Sepmin }}$; where $0<=$ Severity $<=1$. 
Severity could be 1 in the case when both aircraft are at the same point in the horizontal plane (although they could be properly separated vertically) or in the case when both aircraft are at the same altitude (however they could be properly separated horizontally).

Results of PLoS duration analysis show that majority of PLoSs are short, up to $30 \mathrm{sec}$ (roughly two-thirds, i.e. 372 cases), while almost $90 \%$ do not last more than 1 min. Results of severity analysis show that in $80 \%$ of cases severity is 1 , which means that both aircraft were at either the same flight level but not in the same point in the horizontal plane or at the same point in the horizontal plane but at different flight levels.

\subsubsection{Horizontal and vertical separation at closest point of approach}

The results of the distribution of minimum vertical separation at the closest point of approach (CPA) show that almost $80 \%$ of PLoSs are at the same flight level or are separated vertically up to $100 \mathrm{ft}$. The results of horizontal distribution show that roughly $50 \%$ of PLoSs have breach of less than $3 \mathrm{NM}$.

\subsection{Complexity and safety per flight level}

Both complexity and conflict risk can change with flight level. The distribution of an average daily complexity and average daily number of PLoS per FLs is shown on Figure 3.

The highest average values of complexity are on higher altitudes (FL350 to FL380) which correspond to the level used for en route cruise. Somewhat increased values of complexity could be also seen between FL220 and FL240 (corresponds to situations in which flights are entering or leaving lower airspace), however, the number of PLoSs is not increased at this level band.

Distributions of average daily complexity and average daily number of PLoS per FL are similar, with lower values during winter days (Figure 3). Additionally, it can be concluded that traffic demand is influencing higher complexity values; moreover, the number of PLoSs evidently correlates with higher complexity values (Figure 3 ).

Figure 3 shows that in the summer period increase in the number of PLoS at high complexity altitudes is of higher magnitude than in winter months. This could be related to the fact that summer traffic is less predictable (due to the existence of increased number of charter flights and summer destinations traffic).

\section{Conclusion}

Air traffic performance of the European air traffic system depends on traffic demand but also on airspace structure and its traffic distribution. These structural and flow characteristics influence airspace complexity, which can affect controller workload and influence the probability of safety occurrence.

An investigation is performed on FABEC airspace in Europe, based on 2 weeks of realised traffic during summer and winter of 2017, with aim to answer several questions: How changes in traffic demand influence complexity and conflict risk? Is there any correlation between traffic demand, conflict risk and complexity? Are there any differences between seasons? 

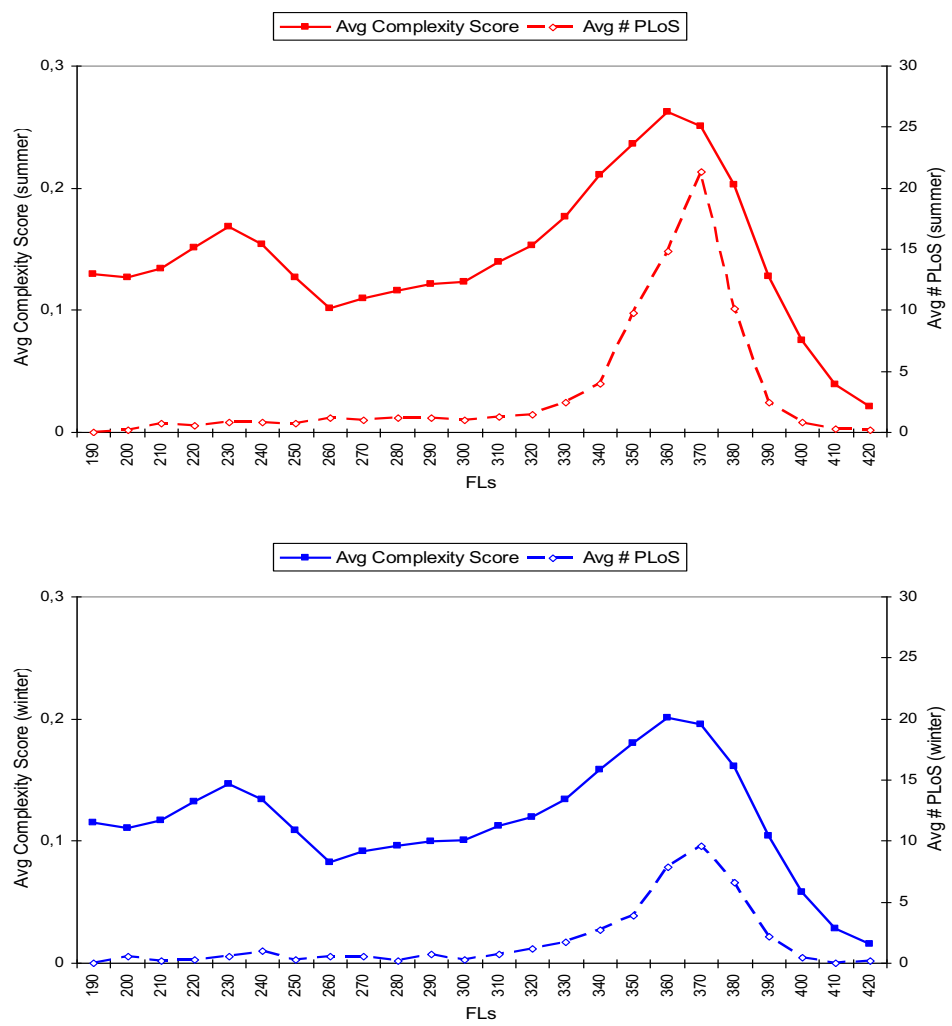

Figure. 3. Distribution of complexity and the number of PLoS per FL (red line - summer/blue line winter)

Daily fluctuations of both complexity and safety indicators follow a similar pattern throughout the week in both summer and winter. Analysis of complexity parameters shows that overall complexity score is mainly influenced by changes in adjusted density which show that interactions are not only related with the traffic volume but also with how this traffic is dispersed in airspace.

The changes in the number of PLoS and conflict risk do not follow strictly the changes in daily traffic demand, and the numbers of PLoS and the conflict risk are lower in winter. This could be related to the fact that traffic demand is lower in winter months and that traffic is more predictable.

Strong correlations were found between traffic demand, safety and complexity indicators. These findings could lead to conclusion that with increase in traffic, one can expect the higher complexity, which in turn influences the number of PLoS and conflict risk. In other words, this means that ATCo task load will increase, leading to a higher ATCo workload.

Both complexity and conflict risk can change with flight level. The highest average values of complexity and number of PLoS are on higher altitudes (FL350 to FL380) which correspond to the level used for en route cruising. Increase in number of PLoS at these altitudes is higher, in relation to increase in complexity, during summer. This could be related to the fact that summer traffic is less predictable (due to existence of increased number of charter flights and summer destinations traffic). 
In a conclusion, this small-scale analysis showed that changes in traffic demand do influence complexity and safety performance (both in terms of the number of PLoS and conflict risk). Moreover, this analysis set a benchmark for future monitoring of safety and operational performance after FRA implementation at FABEC airspace. Further analysis should investigate whether dispersion of traffic after FRA implementation is enough to create complexity decrease and whether change in complexity have not compromised safety and ATCo workload. Moreover, analysis could increase credibility by considering traffic flows, sectors, types of flights (charter, low cost, business, etc.) and vertical profiles of flights.

\section{References}

1. Seven-year Forecast February 2019. EUROCONTROL, Brussels, Belgium, 2019.

2. S. Aneeka, Z. Zhong, "NOX and $\mathrm{CO} 2$ emissions from current air traffic in ASEAN region and benefits of free route airspace implementation". Journal of Applied and Physical Sciences. 2016; 2(2):32-36. DOI: 10.20474/japs-2.2.1

3. Functional airspace blocks. European Commission, Brussels, Belgium, 2019. Available: https://ec.europa.eu/transport/modes/air/single-european-sky/functional-airspaceblocks-fabs_en [Accessed 24 July, 2019].

4. F. Netjasov, M. Janic, V. Tosic, "Developing a generic metric of terminal airspace traffic complexity". Transportmetrica. 2011; 7(5):369-394. DOI: 10.1080/18128602.2010.505590

5. Complexity Metrics for ANSP Benchmarking Analysis. EUROCONTROL, Brussels, Belgium, 2006.

6. T. Pejovic, A. Lazarovski, "Cross border Free Route Airspace implementation performance overview methodology". In: Proceedings of 23rd World Conference of the Air Transport Research Society (ATRS); 2019; Amsterdam, The Netherlands

7. CIRCULAR 319 - A Unified Framework for Collision Risk Modelling in Support of the Manual on Airspace Planning Methodology for the Determination of Separation Minima (Doc 9689), International Civil Aviation Organization, Montreal, Canada, 2009.

8. F. Netjasov, "Framework for airspace planning and design based on conflict risk assessment, Part 2: Conflict risk assessment model for airspace tactical planning". Transportation Research Part C. 2012; 24:213-226. DOI: 10.1016/j.trc.2012.03.003

9. F. Netjasov, D. Crnogorac, G. Pavlovic, "Potential safety occurrences as indicators of air traffic management safety performance: A network based simulation model". Transportation Research Part C. 2019; 102:490-508. DOI: 10.1016/j.trc.2019.03.026

10. Functional Airspace Block Europe Central (FABEC). Available: https://www.fabec.eu/ [Accessed 24 July, 2019]

11. NEST (Network Strategy Tool) User Manual. EUROCONTROL, Brussels, Belgium, 2017.

Fedja Netjasov's work was conducted with support from the Project number 36033 commissioned by the Ministry of Education, Science and Technological development of the Republic of Serbia. 